\title{
Detection of chloramphenicol resistance in Haemophilus influenzae
}

\author{
J J S Snell, S F Perry, D F J Brown
}

\begin{abstract}
An average of $358(13 \%)$ of United Kingdom laboratories failed to detect resistance to chloramphenicol in strains of Haemophilus influenzae distributed by the United Kingdom National External Quality Assessment Scheme for Microbiology. Clear associations between methods used and results obtained show that error rates are minimised by the use of low content $(10 \mu \mathrm{g})$ disc and a sensitive control strain of $\boldsymbol{H}$ influenzae.
\end{abstract}

\section{Methods and Results}

Between September 1985 and March 1990, 12 pure cultures of chloramphenicol resistant Haemophilus influenzae (minimum inhibitory concentrations from 8 to $16 \mathrm{mg} / \mathrm{l}$ ) were distributed as part of the antimicrobial susceptibility testing programme of the United Kingdom National External Quality Assessment Scheme for Microbiology (NEQAS). ${ }^{1}$ Participants were asked to test susceptibility to several named antimicrobial agents including chloramphenicol. An average number of 358 laboratories reported results for chloramphenicol and the percentage incorrectly reporting results as sensitive ranged from $4 \%$ to $21 \%$ (mean $13 \%$ ), depending on the strain. Of the 229 participants examining all 12 cultures, 110 correctly reported resistance to chloramphenicol in all 12. Of the 119 incorrectly reporting one or more cultures as sensitive to chloramphenicol, 53 made this error with one culture, 28 with two, 14 with three, 12 with four, five with five, three with six, one with seven, two with nine and one with 10.

An analysis of information on methods used by participants in September 1985 showed that there was an association between results obtained with two chloramphenicol resistant strains, and both the chloramphenicol content of discs and the use and species of control organisms. ${ }^{2}$ A recent analysis comparing results obtained with six chloramphenicol resistant strains distributed in March 1990 with details of methodology collected concurrently showed the same associations (table). Laboratories using discs containing $10 \mu \mathrm{g}$ of chloramphenicol or less made proportionally fewer errors than those using discs containing more than $10 \mu \mathrm{g}\left(\chi^{2} 126.11 ; \mathrm{p}<0.001\right)$. The probable reasons for this association are the better discrimination in zone sizes between sensitive and resistant strains obtained with low antimicrobial content discs and the larger zone sizes produced by high content discs with resistant strains. ${ }^{3}$ Laboratories using $H$ influenzae as a control strain made proportionally fewer errors than those using the Oxford strain of Staphylococcus aureus ( $\chi^{2}$ $162.74 ; \mathrm{p}<0.001)$ and those not using controls $\left(\chi^{2} 59.69 ; p<0.001\right)$. Laboratories using the Oxford Staphylococcus made proportionally more errors than those not using controls $\left(\chi^{2} 5.88 ; p<0.05\right)$.

\section{Discussion}

In view of the possible serious clinical consequences of failure to detect resistance to chloramphenicol in $H$ influenzae the high error rate shown by this series and the tendency of some laboratories to repeat errors must be cause for concern. Considerable reduction in these error rates could be achieved by simple changes in methodology. The magnitude of the potential for improvement is apparent from the fact that with the six strains distributed in March 1990, the average error rates (failure to detect resistance to chloramphenicol) were as follows: $42 \cdot 8 \%$ for the subset of 15 laboratories using high content discs $(>10 \mu \mathrm{g})$ and not using $H$ influenzae as a control organism; $1 \cdot 2 \%$ for the subset of 159 laboratories using low content discs and $H$ influenzae as a control. Most laboratories in the United Kingdom use either Stokes' or the comparative method of disc susceptibility testing, ${ }^{4}$ or modifications thereof, and these findings may only be relevant to these methods. For most United Kingdom users of disc methods, however, these results speak for themselves and we urge laboratories to adopt the use of low content discs $(10 \mu \mathrm{g})$ and an $H$ influenzae control ( $H$ influenzae NCTC 11931 is available ${ }^{2}$ ).

1 Snell JJS, De Mello JV, Gardner PS. The United Kingdom national microbiological quality assessment scheme. $J$ Clin Pathol 1982;35:82-93.

2 Snell JJS, Brown DFJ; Phua TJ. Antimicrobial susceptibility testing of Haemophilus influenzae: trial organised as part of United Kingdom national external quality assessment scheme for microbiology. J Clin Pathol 1986;39:1006-12.

3 Walker CW, Brown DFJ. The reliability of methods for detecting chloramphenicol resistance in Haemophilus influenzae. $J$ Antimicrob Chemother 1988;22:905-10.

4 Stokes EJ, Waterworth PM. Antibiotic sensitivity tests by diffusion methods. ACP Broadsheet 55. London: BMA/ ACP, 1972.
Controls used Ho control use

Oxford Staphylococcus

177
40
80 